\title{
Visual outcome in Japanese patients with Acanthamoeba keratitis
}

K Yamazoe ${ }^{1,2}$, Y Yamamoto' ${ }^{1}$ S Shimazaki-Den ${ }^{1}$

K Yamazoe ${ }^{1,2}$, Y Yam
and J Shimazaki ${ }^{1,3}$

Abstract

Purpose To identify prognostic factors affecting visual outcome in Acanthamoeba keratitis (AK) treated with topical chlorhexidine gluconate (CHG). Methods A total of 35 eyes in 34 patients with AK were treated with $0.02 \%$ topical CHG. Patients were divided into two groups according to the final visual outcome: Group 1, final visual acuity (VA) of 20/25 or greater (22 eyes); Group 2, less than 20/25 (13 eyes). We compared these groups and evaluated the effectiveness of topical CHG compared with outcomes in previous reports. Results Ring infiltrate was observed more often in Group 2 (4.5\% vs 61.5\%, OR 33.6, 95\% confidence interval (CI) 3.4-333.9, $P<0.01)$. The duration between onset and diagnosis of AK was significantly longer (24.9 days vs 48.4 days, OR 1.03, 95\% CI 1.00-1.06, $P=0.04)$ and VA at initial examination (log MAR) significantly lower (0.47 vs 1.59, OR 25.5, 95\% CI 3.4-186.7, $P<0.01$ ) in Group 2 (visual outcome $<20 / 25$ ). Multivariate analysis revealed that only VA at initial examination was independently associated with worse visual outcome (adjusted OR 24.5, 95\% CI 1.9-312.6, $\mathrm{P}=0.01$ ). Seventeen $(85.0 \%)$ of the 20 eyes diagnosed within 1 month and $24(82.8 \%)$ of 29 eyes diagnosed within 2 months achieved a VA of $20 / 40$ or greater.

Conclusion VA at initial examination was the most predictive factors for final visual outcome in AK. Topical CHG was comparably effective to other treatments, including polyhexamethyl biguanide and propamidine isethionate.

Eye (2012) 26, 517-522; doi:10.1038/eye.2011.366; published online 13 January 2012

Keywords: Acanthamoeba; keratitis; chlorhexidine gluconate; keratoplasty
Introduction

Despite being a relatively rare disease in comparison with other infectious keratitis, Acanthamoeba keratitis (AK) is a potentially blinding disease and has been reported in many countries. ${ }^{1-5}$ However, specific treatment is not established, and there are some differences in the treatment between Western and Asian countries. In Japan, anti-fungal agents, corneal scraping, chlorhexidine gluconate (CHG), or some combination of these therapies are often used to treat AK. However, polyhexamethyl biguanide (PHMB) and propamidine isethionate are often used in Western countries. ${ }^{1,4,6-8}$ Biguanides such as CHG and PHMB are the most effective cysticidal antiamoebics, and are currently used as a firstline treatment for $\mathrm{AK} .{ }^{9}$ Lim et $a l^{10}$ compared the therapeutic outcomes for PHMB and CHG treatment and reported that, although the results were non-significant, $78 \%$ of eyes receiving $\mathrm{PHMB}$ and $86 \%$ of eyes receiving CHG were successfully treated.

In this study, we report therapeutic outcomes and prognostic factors for 35 Japanese patients with AK who were treated with topical CHG. As PHMB is not available in Japan, CHG was used in the present study as the main treatment agent.

Patients and methods

Subjects

We reviewed the medical records of patients who were diagnosed with AK between January 2001 and December 2009 at the Tokyo Dental College, Ichikawa General Hospital, Japan. All patients were treated according to the Hospital's standardised treatment protocol. The patients were divided into two groups based on the final visual outcome, defined as best corrected visual acuity (BCVA) at the time
${ }^{1}$ Department of Ophthalmology, Tokyo Dental College, Chiba, Japan

${ }^{2}$ Department of Ophthalmology, Kameda Medical Center, Chiba, Japan

${ }^{3}$ Department of Ophthalmology, Keio University School of Medicine, Chiba, Japan

Correspondence: K Yamazoe, Department of Ophthalmology, Tokyo Dental College, 5-11-13 Sugano, Ichikawa, Chiba 272-8513, Japan Tel: + 8147322 0151; Fax: +81473226786. E-mail: kyamazoe1528@ yahoo.co.jp

Received: 12 July 2011 Accepted in revised form: 26 November 2011 Published online: 13 January 2012 
of the disappearance of conjunctival congestion or corneal oedema. In patients who underwent keratoplasty, the BCVA before surgery was measured as the final visual outcome, as follows: Group 1 consisted of patients with a final visual acuity (VA) of 20/25 or greater; Group 2 consisted of patients with a final VA of less than 20/25.

This study was conducted in accordance with the tenets of the Helsinki declaration and approval was obtained from ethics committees at our institution. All patients provided written informed consent for treatment with CHG.

\section{Method of diagnosis}

All patients underwent corneal scraping during the initial visit. A diagnosis of AK was based on the identification of positive Acanthamoeba cultures from a corneal scrape on non-nutrient agar with an Escherichia coli overlay, and/or typical clinical characteristics, including radial neuritis, ring infiltrate, and pseudodendritic lesions.

\section{Treatment protocol}

All patients were treated with wide mechanical epithelial debridement during the initial visit and hourly treatment with $0.02 \%$ topical CHG. Debridement was repeated according to clinical course. In 20 eyes, topical antifungal agents were applied hourly, including $0.1 \%$ miconazole nitrate (Florid-F, Mochida, Tokyo, Japan) in 13 eyes, $0.1 \%$ fluconazole (Diflucan, Pfizer Japan, Tokyo, Japan) in six eyes, and $0.1 \%$ micafungin sodium (Funguard, Astellas, Tokyo, Japan) in five eyes. In 25 patients, topical anti-bacterial agents were applied or continued, including 0.5\% Levofloxacin (Cravit, Santen, Osaka, Japan) in 19 eyes, 0.3\% Gatifloxacin (Gatifo, Senju, Osaka, Japan) in 2 eyes, 0.5\% Moxifloxacin (Vigamox, Alcon Japan, Tokyo, Japan) in 2 eyes, $0.5 \%$ Cefmenoxime (Bestron, Senju) in 2 eyes, 0.3\% Ofloxacin (Tarivid, Santen) in 1 eye, and $0.3 \%$ Tosufloxacin (Tosuflo, Nittomedic, Toyama, Japan) in 1 eye. Steroids and acyclovir ointment were discontinued if used by previous doctors. Topical CHG administration was tapered according to the clinical response, as indicated by a reduction in corneal oedema or conjunctival congestion.

\section{Outcome measures}

Parameters of interest included the duration between onset and diagnosis, diagnosis and treatment by previous doctors, BCVA upon initial examination, clinical findings upon initial examination, positive culture rate for Acanthamoeba, and final visual outcome.
These factors were assessed and compared between Groups 1 and 2 to determine factors affecting final visual outcome in AK. We also evaluated effectiveness of treatment, including final visual outcome and rate of need for keratoplasty, and compared these with previous reports.

\section{Statistical analysis}

Statistically significant differences between the two groups were determined using a logistic regression. A $P$-value of $<0.05$ was considered statistically significant. Multivariate logistic regression analyses were performed for variables that were statistically significant in the univariate analyses. All statistical analyses were performed with SSRI software (SSRI, Tokyo, Japan).

\section{Results}

\section{Demographic features}

AK was diagnosed in 35 eyes of 34 patients. Only one patient showed bilateral involvement. The mean age was $29.4 \pm 8.9$ years (range, 16 to 49 years). The proportion of male patients $(n=25)$ was greater than that of female patients $(n=10)$. All patients were referred by local doctors and the mean duration between onset and diagnosis was $33.6 \pm 29.5$ days (range, $1-131$ days). All patients used contact lenses. Data regarding type of contact lens were available for all patients. Of these, 34 patients used soft contact lenses and one patient used hard lenses. Thirty-one patients used disposable contact lenses, 24 of which were used for two weeks at a time; the remainder were used for one day and disposed.

\section{Clinical features}

Microbiological confirmation of disease was present in 20 (57.1\%) of the 35 eyes. The remainder were diagnosed based on clinical features, including radial neuritis $(n=7)$, ring infiltrate $(n=5)$, or pseudodendritic lesions $(n=3)$. The presumed diagnosis upon referral was correct in only one case. The remaining 34 eyes had been misdiagnosed with herpes simplex virus keratitis ( $n=20)$, bacterial ulcer $(n=11)$, keratoconjunctivitis of unknown origin $(n=2)$, or uveitis $(n=1)$. Before the diagnosis of $\mathrm{AK}, 16$ eyes had been treated with corticosteroids, including topical administration in 13 eyes and systemic administration in two eyes. In addition, topical acyclovir ointment $(n=19)$ and topical antibacterial agents $(n=20)$ had been administered. At the initial visit, radial neuritis was observed in 17 (48.6\%) of the 35 eyes. Ring infiltrate was observed in eight $(22.9 \%)$ of the 35 eyes. Other clinical signs included 
pseudodendritic lesions (7 of 35 eyes, 20.0\%), nummular subepithelial infiltrates ( 35 of 35 patients, $100 \%$ ), and dacryoadenitis ( 3 of 35 eyes, 8.6\%). The overall mean BCVA upon initial presentation was 20/155 (range, hand motion to 20/17).

\section{Management and clinical outcomes}

Treatment with $0.02 \%$ topical CHG was initiated in all patients as soon as a clinical or microbiological diagnosis of AK was made. Final BCVA ranged from hand motion to 20/17 (mean, 20/49), and 77.1\% achieved greater than 20/40. Of the 35 eyes, 22 (62.9\%) achieved a final VA of 20/25 or greater (Group 1: mean age, $29.9 \pm 7.6$ years; median follow-up 6.7 months, IQR 5.2-11.8 months) and $13(37.1 \%)$ achieved less than 20/25 (Group 2: mean age, 28.5 \pm 11.0 years; median follow-up 9.5 months, IQR 4.7-42.5 months). Overall, the average duration of treatment was median 101 days (IQR 61-144 days). After treatment with $0.02 \%$ topical $\mathrm{CHG}$, three patients received optical keratoplasty. No eyes required therapeutic keratoplasty. Overall, $18(90.0 \%)$ of 20 patients in whom a diagnosis was reached within 1 month and $26(90.0 \%)$ of 29 patients in whom a diagnosis was reached within 2 months achieved a BCVA of 20/40 or greater after keratoplasty.

The relationship between the base line characteristics, including prior treatment, culture positivity, and clinical features and initial VA was also evaluated with a regression analysis. As a result, the existence of ring infiltrate $(P<0.01)$ and duration before diagnosis $(P<0.01)$ were associated with initial VA.

\section{Keratoplasty}

Optical keratoplasty was performed in three eyes with a VA of less than 20/200. Penetrating keratoplasty (PKP) was performed in two eyes and deep anterior lamellar keratoplasty (DALK) in one eye. All were indicated to improve vision impaired by scarring. VA after keratoplasty was 20/20 in two eyes and 20/25 in one eye. Evolution from VA at presentation to final VA is shown in Table 1. The microbiological cultures of the corneal buttons were negative and there was no evidence of infection in histopathological test.

\section{Factors affecting final visual outcome}

Differences were not observed because of age, gender, diagnosis and treatment by previous doctors, or culturepositive rates between the two groups (Table 2).

Corticosteroids were used more often in Group 2 (OR $4.82,95 \%$ confidence interval (CI) $1.1-21.2, P=0.04)$. Ring infiltrate was observed more often in Group 2 (OR 33.6, 95\% CI 3.4-333.9, $P<0.01)$. Symptom duration before diagnosis was significantly longer in Group 2 (48.4 \pm 36.3 days; range, 10-131 days) than in Group 1 (24.9 \pm 21.1 days; range, 1 to 77 days; OR 1.03, 95\% CI 1.00-1.06, $P=0.04)$. VA ( $\log$ MAR) upon first examination was significantly worse in Group 2 ( $0.47 \pm 0.42$ vs $1.59 \pm 0.79$; OR 25.5, 95\% CI 3.4-186.7, $P<0.01$ ). Eyes with VA (log MAR) at first examination worse than 0.50 were more often in Group 2 (OR 32.0, 95\% CI 3.4-186.7). Twelve $(60.0 \%)$ of the 20 eyes treated with antifungal agents were in Group 1 and ten $(66.6 \%)$ of 15 treated without antifungal agents were in Group 1 (OR 1.33, 95\% CI $0.33-5.39, P=0.69$ ). The relationship between duration before diagnosis and final BCVA was analysed. In all, $15(75.0 \%)$ of 20 eyes diagnosed within 1 month achieved a BCVA of $20 / 25$ or greater. However, one (16.7\%) of the six eyes diagnosed after at least 2 months achieved a BCVA of $20 / 25$ or greater.

Multivariate logistic regression analyses were performed for variables that were statistically significant in the univariate analyses, and only VA at initial examination remained significant (adjusted OR 24.5, 95\% CI 1.9-312.6, $P=0.01)$.

\section{Side effects of $\mathrm{CHG}$}

No severe adverse reactions to $\mathrm{CHG}$, such as toxicity, exacerbation of inflammation, oedema, chronic corneal ulcers, or opacification, were observed in any patient.

\section{Discussion}

Although previous studies have investigated the treatment of $\mathrm{AK}$ with $\mathrm{CHG},{ }^{10,11}$ we believe this study to be one of the largest studies to investigate visual outcomes in Asia. We treated all patients with topical CHG following a standardised protocol, which was different from similarly designed studies. ${ }^{12}$

Table 1 Pre- and postoperative visual outcomes in three cases requiring keratoplasty

\begin{tabular}{lccccccc}
\hline Age & Gender & DBD (days) & VA at the initial examination & VA before KP & Type of KP & VA after KP & POP (months) \\
\hline 30 & Female & 39 & $20 / 40$ & $20 / 500$ & PKP & $20 / 29$ & 46.2 \\
15 & Male & 25 & $20 / 500$ & $20 / 133$ & DALK & $20 / 20$ & 22.8 \\
22 & Male & 65 & $20 / 400$ & HM & PKP & $20 / 20$ & 15.0 \\
\hline
\end{tabular}

Abbreviations: DBD, duration before diagnosis; VA, visual acuity; HM, hand motion; KP, keratoplasty; PKP, penetrating keratoplasty; DALK, deep anterior lamellar keratoplasty; POP, postoperative observation period. 
Table 2 Comparison of clinical characteristics between Group 1 and Group 2

\begin{tabular}{|c|c|c|c|c|}
\hline & Group $1(V A \geq 20 / 25)(\%)$ & Group $2(V A<20 / 25)(\%)$ & OR $(95 \% C I)$ & P-value \\
\hline \multicolumn{5}{|l|}{ Gender } \\
\hline Male & $17(77.3)$ & $8(61.5)$ & $0.47(0.11-2.10)$ & 0.32 \\
\hline Female & $5(22.7)$ & $5(38.4)$ & 1.00 & \\
\hline \multicolumn{5}{|l|}{ Treatment before diagnosis } \\
\hline Steroid & $7(31.8)$ & $9(69.2)$ & $4.82(1.1-21.2)$ & 0.04 \\
\hline Topical acyclovir ointment & $12(54.5)$ & $7(53.8)$ & $0.97(0.25-3.85)$ & 0.97 \\
\hline Antibacterial treatment ${ }^{a}$ & $13(59.1)$ & $7(53.8)$ & $0.80(0.20-3.21)$ & 0.76 \\
\hline \multicolumn{5}{|l|}{ Clinical features } \\
\hline Radial neuritis & $13(59.1)$ & $4(30.8)$ & $0.31(0.07-1.31)$ & 0.11 \\
\hline Ring infiltrates & $1(4.5)$ & $8(61.5)$ & $33.6(3.4-333.9)$ & $<0.01$ \\
\hline Pseudodendritic lesions & $6(27.3)$ & $1(7.7)$ & $0.22(0.02-2.10)$ & 0.19 \\
\hline Dacryoadenitis & $2(9.1)$ & $1(7.7)$ & $0.83(0.07-10.20)$ & 0.89 \\
\hline Culture positive & $14(63.6)$ & $6(46.2)$ & $0.49(0.12-1.97)$ & 0.31 \\
\hline \multicolumn{5}{|l|}{ Treatment } \\
\hline CHG without anti-fungal agents & $10(66.6)$ & $5(33.3)$ & 1.00 & - \\
\hline \multirow[t]{2}{*}{ CHG with anti-fungal agents } & $12(60.0)$ & $8(40.0)$ & $1.33(0.33-5.39)$ & 0.69 \\
\hline & Mean \pm SD & Mean \pm SD & & \\
\hline Age & $29.9 \pm 7.6$ & $28.5 \pm 11.0$ & $0.98(0.91-1.06)$ & 0.64 \\
\hline Duration before diagnosis (days) & $24.9 \pm 21.1$ & $48.4 \pm 36.3$ & $1.03(1.00-1.06)$ & 0.04 \\
\hline Duration of treatment (days) & $116.5 \pm 107.2$ & $154.5 \pm 102.8$ & $1.00(0.99-1.01)$ & 0.31 \\
\hline$V A$ at initial examination $(\log M A R)$ & $0.47 \pm 0.42$ & $1.59 \pm 0.79$ & $25.5(3.4-186.7)$ & $<0.01$ \\
\hline$<0.50$ & 16 & 6 & 1.00 & \\
\hline$\geq 0.50$ & 1 & 12 & $32.0(3.4-302.2)$ & $<0.01$ \\
\hline
\end{tabular}

Abbreviations: VA, visual acuity; $\mathrm{CHG}$, chlorhexidine gluconate.

${ }^{\mathrm{a}} 0.5 \%$ Levofloxacin (13), 0.3\% Gatifloxacin (2), 0.5\% Moxifloxacin (2), 0.5\% Cefmenoxime (2), 0.3\% Ofloxacin (1), 0.3\% Tosufloxacin (1).

Bold values indicate $P<0.05$.

In the present study, the presence of AK was confirmed through microbiological evidence in $57.1 \%$ of patients. This rate of positivity was similar to those in earlier reports. ${ }^{1,3,6,7}$ Herpes simplex virus keratitis was the most common misdiagnosis, ${ }^{4}$ which may reflect the similarity between this disease and typical nonspecific findings early in the course of $\mathrm{AK}$, including epithelial microabrasion, irregularities, pseudodendritic lesions, and subepithelial infiltrates. All patients used contact lenses, and the majority used soft disposable lenses. It has been previously reported that contact lens use is a major risk factor for AK. ${ }^{1,2,4,8}$ Although some reports have suggested that the use of daily-disposable lenses poses only a low risk of $\mathrm{AK}^{5}$ seven of the $\mathrm{AK}$ patients examined in our study used this type of lens. However, it is difficult to use our results to generalise the level of risk of $\mathrm{AK}$ as the usage of daily-type contact lenses differs greatly among countries. Nevertheless, it is notable that the use of daily-disposable contact lenses does not preclude the occurrence of Acanthamoeba infections.

Symptom duration before diagnosis and VA upon initial examination were strongly associated with the final visual outcome. Previous studies have suggested that an earlier diagnosis results in more favourable outcomes, ${ }^{1,3,6,13-15}$ and our results support this proposition. However, Tu et $a l^{12}$ suggested that symptom duration was not a strong predictor of final visual outcome when compared with corneal disease stage at the initial visit. The authors also suggested that deep stromal involvement or the presence of a ring infiltrate at first visit was independently associated with poor visual outcomes. In our study, ring infiltrates were only observed in patients with poor visual outcome. The severity of disease at the initial visit, leading to poor VA at the initial examination, and the presence of a ring infiltrate were predictive factors for poor final visual outcome in AK. Although radial neuritis has been associated with good results in some studies, ${ }^{4}$ no statistically significant difference was observed in our study. Radial neuritis was useful for diagnosis, but was not a reliable predictor of final visual outcome.

We compared visual outcomes in our study with outcomes achieved in other countries where a combination therapy of PHMB and propamidine isethionate was administered. Duguid et $a l^{7}$ achieved a 
BCVA of $20 / 40$ or greater in $91 \%$ of the early treatment group (diagnosis within 28 days) and $71 \%$ of the intermediate group (diagnosis within 28 days to 2 months). Butler $e t a l^{6}$ carried out a recent 6-year review of AK among the population of New South Wales, Australia, and reported that $75 \%$ achieved a BCVA of 20/40 or greater. However, BCVA was determined after surgical intervention in seven cases in their study. Their mean interval to diagnosis was 26.6 days. In our study, a BCVA of $20 / 40$ or greater was achieved in $77.1 \%$ of eyes. After keratoplasty, $85.7 \%$ achieved a BCVA of 20/40 or greater. Allowing for the fact that the durations of these two studies were shorter than the present study, our results could be considered comparable. In fact, 18 $(90.0 \%)$ of 20 patients in whom a diagnosis was reached within 1 month and $26(90.0 \%)$ of 29 patients in whom a diagnosis was reached within 2 months achieved a BCVA of 20/40 or greater after keratoplasty. In previous studies, wide mechanical epithelial debridement was reported to be effective due to the reduction of infectious organisms and improvement in topical drug penetration. ${ }^{16}$ Debridement was performed upon the first visit for all of our patients and then repeated according to clinical course. Debridement was not performed, however, in the two earlier studies discussed above. Therefore, drug therapy combined with scraping may have improved the final BCVA. Although a simple comparison between our results and previous reports is difficult due to the different condition of patients, we thought that our results, including final visual outcome after adjustment for the duration before treatment and that no eyes required therapeutic keratoplasty could be comparable. In our study, additional antifungal agents did not affect the final visual outcome. Although further study will be necessary, treatment with only CHG might be as effective as combination treatment.

Keratoplasty was performed in three $(8.6 \%)$ of the 35 patients in our study. In a study from Singapore, 15 $(35.7 \%)$ of the 42 patients under study required corneal grafting, with 11 undergoing therapeutic DALK, two undergoing optical PKP, one undergoing optical DALK, and one undergoing therapeutic PKP. ${ }^{5}$ In a study from England, 40 (16.5\%) of 243 patients underwent surgery. ${ }^{1}$ Illingwort et $a l^{14}$ reported that PKP was performed in nine $(39.1 \%)$ out of 23 eyes. In our study, the rate of patients requiring keratoplasty was lower and optical keratoplasty was performed in all three cases. Kitzmann et $a l^{17}$ reported that optical keratoplasty performed after resolution of active keratitis was associated with an excellent prognosis for both graft survival and visual outcome. In our cases, a median VA of 20/23 was obtained after optical keratoplasty, which is comparable to results reported by Kitzmann et al ${ }^{17}$ (20/25) and Ficker et al $^{18}$ (20/30). These results may have been influenced by the effect of $\mathrm{CHG}$ or combination treatment with corneal scraping or antifungal agents.

One of the limitations of our study is that 15 of the 35 cases in this series were diagnosed clinically, in the face of a negative culture. Although the rate was similar to those discussed in previous reports, ${ }^{1,3,6,7}$ fungal or bacterial infections were not completely excluded in culture negative patients as radial neuritis and ring infiltrates are not pathognomonic for AK, and antifungal or antibacterial medications could affect the results in these cases.

In conclusion, the most important factors affecting prognosis in AK were the involvement of stromal tissue and the interval between onset of symptoms and the start of effective therapy. Treatment with topical CHG seems to be as effective as combination therapy with $\mathrm{PHMB}$ and propamidine isethionate.

\section{Summary}

\section{Conflict of interest}

The authors declare no conflict of interest.

\section{Acknowledgements}

The English in this document has been checked by at least two professional editors, both native speakers of English. For a certificate, please see: http://www. textcheck.com/certificate/WH14rx.

\section{References}

1 Radford CF, Lehmann OJ, Dart JK. Acanthamoeba keratitis: multicentre survey in England 1992-6. National Acanthamoeba Keratitis Study Group. Br J Ophthalmol 1998; 82: 1387-1392.

2 Joslin CE, Tu EY, McMahon TT, Passaro DJ, Stayner LT, Sugar J. Epidemiological characteristics of a Chicago-area Acanthamoeba keratitis outbreak. Am J Ophthalmol 2006; 142: $212-217$

3 Sun X, Zhang Y, Li R, Wang Z, Luo S, Gao M et al. Acanthamoeba keratitis: clinical characteristics and management. Ophthalmology 2006; 113: 412-416. 
4 Thebpatiphat N, Hammersmith KM, Rocha FN, Rapuano CJ, Ayres BD, Laibson PR et al. Acanthamoeba keratitis: a parasite on the rise. Cornea 2007; 26 : 701-706.

5 Por YM, Mehta JS, Chua JL, Koh TH, Khor WB, Fong AC et al. Acanthamoeba keratitis associated with contact lens wear in Singapore. Am J Ophthalmol 2009; 148: 7.e2-12.e2.

6 Butler TK, Males JJ, Robinson LP, Wechsler AW, Sutton GL, Cheng J et al. Six-year review of Acanthamoeba keratitis in New South Wales, Australia: 1997-2002. Clin Experiment Ophthalmol 2005; 33: 41-46.

7 Duguid IG, Dart JK, Morlet N, Allan BD, Matheson M, Ficker $\mathrm{L}$ et al. Outcome of acanthamoeba keratitis treated with polyhexamethyl biguanide and propamidine. Ophthalmology 1997; 104: 1587-1592.

8 Radford CF, Minassian DC, Dart JK. Acnthamoeba keratitis in England and Wales: incidence, outcome, and risk factors. Br J Ophthalmol 2002; 86: 536-542.

9 Dart JK, Saw VP, Kilvington S. Acanthamoeba keratitis: diagnosis and treatment update 2009. Am J Ophthalmol 2009; 148: 487.e2-499.e2.

10 Lim N, Goh D, Bunce C, Xing W, Fraenkel G, Poole TR et al. Comparison of polyhexamethylene biguanide and chlorhexidine as monotherapy agents in the treatment of Acanthamoeba keratitis. Am J Ophthalmol 2008; 145: 130-135.

11 Kosrirukvongs P, Wanachiwanawin D, Visvesvara GS. Treatment of acanthamoeba keratitis with chlorhexidine. Ophthalmology 1999; 106: 798-802.

12 Tu EY, Joslin CE, Sugar J, Schoff ME, Booton GC. Prognostic factors affecting visual outcome in Acanthamoeba keratitis. Ophthalmology 2008; 115: 1998-2003.

13 Claerhout I, Goegebuer A, Van Den Broecke C, Kestelyn P. Delay in diagnosis and outcome of Acanthamoeba keratitis. Graefes Arch Clin Exp Ophthalmol 2004; 242: 648-653.

14 Illingworth CD, Cook SD, Karabatsas CH, Easty DL. Acanthamoeba keratitis: risk factors and outcome. $\mathrm{Br} J$ Ophthalmol 1995; 79: 1078-1082.

15 Bacon AS, Dart JK, Ficker LA, Matheson MM, Wright P. Acanthamoeba keratitis. The value of early diagnosis. Ophthalmology 1993; 100: 1238-1243.

16 Brooks Jr JG, Coster DJ, Badenoch PR. Acanthamoeba keratitis. Resolution after epithelial debridement. Cornea 1994; 13: 186-189.

17 Kitzmann AS, Goins KM, Sutphin JE, Wagoner MD. Keratoplasty for treatment of Acanthamoeba keratitis. Ophthalmology 2009; 116: 864-869.

18 Ficker LA, Kirkness C, Wright P. Prognosis for keratoplasty in Acanthamoeba keratitis. Ophthalmology 1993; 100: 105-110. 